$\$$ Research Square

\title{
Unravelling hierarchical levels of structure in lipid membranes
}

\author{
Alexandre Blanco-González \\ University of Santiago de Compostela \\ Rebeca Garcia Fandiño \\ USC \\ Angel Piñeiro ( $\nabla$ Angel.Pineiro@usc.es ) \\ Dept. Fisica Aplicada, Universidad de Santiago de Compostela
}

\section{Article}

Keywords: Lipid bilayer, cell membrane, hierarchical levels of structure, molecular 29 dynamics simulations

Posted Date: February 4th, 2022

DOI: https://doi.org/10.21203/rs.3.rs-1287323/v1

License: (c) (i) This work is licensed under a Creative Commons Attribution 4.0 International License. Read Full License 


\section{Unravelling hierarchical levels of structure in lipid membranes}

5

$6 \quad{ }^{1}$ Departamento de Física Aplicada, Facultade de Física, Universidade de Santiago

de Compostela, E-15782 Santiago de Compostela, Spain. Departamento de Química

Orgánica, Center for Research in Biological Chemistry and Molecular Materials,

Universidade de Santiago de Compostela, Campus Vida s/n, E-15782 Santiago de Compostela, Spain. ${ }^{3}$ MD.USE Innovations SL, Edificio Emprendia, 15782 Santiago de Compostela, Spain.

*Corresponding authors: rebeca.garcia.fandino@usc.es, Angel.Pineiro@usc.es

\section{Abstract:}

In analogy with the hierarchical levels typically used to describe the structure of nucleic acids or proteins, and keeping in mind that lipid bilayers are not just mere envelopers for biological material but directly responsible for many important functions of life, we propose and discuss here how membrane models can also be interpreted in terms of different hierarchies in their structure. Namely, lipid composition, interaction between leaflets, existence and interaction of domains arising from the coordinate behavior of lipids and their properties, plus the manifest and specific perturbation of the lipid organization around macromolecules embedded in a membrane are hereby used to define the primary, secondary, tertiary and quaternary structures, respectively. Molecular Dynamics simulations are used to illustrate this proposal. The new paradigm arising from such description is expected to have significant consequences on 
26 deciphering the underlying factors governing membranes and their interactions with

27 other molecules.

28 Keywords: Lipid bilayer, cell membrane, hierarchical levels of structure, molecular 29 dynamics simulations.

30

31 


\section{1.- Introduction}

33 What Rosalind Franklin, James Watson and Francis Crick experienced when they first

34 got a glimpse of the DNA 3D structure ${ }^{1,2}$, or when John Kendrew captured what was coming out of myoglobin RX into his sausage model ${ }^{3,4}$, was surely comparable to what a 20 diopters-myopic person feels when putting on glasses for the first time. Those events changed the logic of biology because the way in which we currently view DNA or proteins affects how we think about their function. This has a significant impact on hypotheses development, experimental design, and data interpretation.

The three-dimensional structure of large biological molecules such as proteins and nucleic acids is complex and confusing to the naked eye. It is not easy to describe them accurately using just words. Because of this, it is convenient to introduce new concepts. A series of hierarchical levels -typically referred as primary, secondary, tertiary, and quaternary structures- are commonly employed to describe biological macromolecules. These different "organization levels" exhibit regularity and predictability for natural phenomena represented in diverse ways, thus facilitating scientific inquiry and hierarchical causal explanations of different biological processes.

In proteins and nucleic acids, the primary structure is associated to the covalent chemical structure. It usually consists of linear arrays of covalently bound building blocks with a common scaffold and a variety of chemical substitutions. The number of different building blocks is restricted to five in typical nucleic acids (including RNA), and to twenty in proteins. Thus, the primary structure can be specified by the linear order, or sequence, of these building blocks. The secondary structure refers to regular patterns of interaction between adjacent or facing residues. The most illustrative examples are alphahelices or beta-sheets observed in many proteins and double helices found in virtually all 
nucleic acids. The tertiary structure refers to the location of the atoms in the three-

57 dimensional space, taking into consideration geometrical and steric constraints. It usually provides the starting point for studies that attempt to correlate structure and function. In proteins it comes from the folding of the secondary structure into distinct arrangements known as domains, whereas the tertiary arrangement of DNA's double helix in space

61 includes B-DNA, A-DNA, and Z-DNA. Quaternary structure describes the assembly of 62 individual molecular units into more complex arrays. The simplest example of quaternary structure is a protein that consists of multiple subunits, which are not connected to each other by covalent bonds and may be identical or different. In nucleic acids, it refers to interactions with other molecules, for example in the form of chromatin which leads to its interactions with the histone small proteins.

67 This hierarchical representation in four structural layers of organization is extremely robust and well established empirically for proteins and nucleic acids but it quickly loses its significance when applied throughout the whole spectrum of biological entities. Different macromolecules and supramolecular structures, such as polysaccharides and lipid membranes, exhibit clear three-dimensional shapes and are built out of smaller subunits. However, different levels of structure have not been defined in these cases. Polysaccharides are quite similar to proteins in that they are chains of repeated building blocks (monosaccharides) that acquire well-defined three-dimensional topologies. The case of cell membranes requires a more detailed explanation. According to the fluid mosaic model proposed by Singer and Nicolson in $1972^{5}$, the cellular plasma membrane is a two-dimensional liquid, in which lipid and protein molecules are mixed like a mosaic and undergo thermal diffusion. This model is still widely accepted by the research community. Under this approach all the molecules are expected to be homogeneously distributed throughout the plasma membrane, undergoing simple Brownian diffusion. 
81 However, an increasing number of evidence showing that this is not the case is being

82 found $^{6}$. Like proteins, nucleic acids, and even sugars, lipid membranes can be also 83 described using a hierarchical organization in different levels of structure. The new 84 paradigm arising from such description is expected to help to understand the underlying 85 factors governing membranes and their interactions with other molecules. This is 86 especially important since lipid membranes do constitute one of the most important parts 87 of any living cell, acting as the main barrier between the cytoplasm and its surrounding environment ${ }^{7}$. Their function is not limited to a mere division between the inside and the outside of cells, but it is extended to a wide range of processes, such as serving as anchor 90 points and modulators for signaling ${ }^{8,9}$ and transport ${ }^{9,10}$ proteins, or playing a key role in 91 cell motility ${ }^{9,11}$. Lipid membranes are also known to be subject to substantial attacks and 92 changes upon disease related processes. For instance, some families of pathogens such as enveloped viruses -HIV and SARS-CoV-2, among many others- are known to enter a 94 cell by fusing their membranes with the host's ${ }^{12,13}$. Once internalized and just before the viral reproduction cycle is completed, they leave the cell by selectively ripping apart ${ }^{14}$ the 96 infected cell's membrane, making it their own and leaving the host's cell heavily modified 97 in its lipidic composition. This modification is hypothesized to sometimes be able to trigger an autoimmune response in the diseased host leading to chronic inflammation ${ }^{15}$, 99 which might have fatal consequences. On the other hand, cancerous processes are also 100 known to display significant alterations on the membranes of the affected tissues ${ }^{16,17}$.

101 While healthy mammalian somatic cells present an asymmetry in their composition depending on the leaflet, leaving most of the negatively charged lipids towards the

103 cytoplasm; cancer cells tend to lose this property providing them with negative charges

104 on their exterior, a characteristic that share with bacterial cells ${ }^{18,19}$. All of this illustrates

105 the important role that lipid membranes play as targets both to invasive pathogens and to 
106 potential therapies for diseases derived from such processes. It is also reasonable to think

107 that they play a role in some chronic autoimmune responses ${ }^{20,21}$. Therefore, a proper

108 understanding of the underlying factors governing membranes and their interactions with

109 different species is mandatory to obtain rational criteria from which then derive effective

110 and efficient solutions to the problems that they are involved in. Characterizing living

111 membranes is not easy, as they are densely packed fluids with literally thousands of

112 different components, and their compositions vary greatly depending on the type or

113 source of the cell. Even with much simpler models, empirical study can be cumbersome

114 and there are great difficulties in reaching the resolution needed to get to the atomic or

115 quasi-atomic levels at which some important processes happen. Therefore, computational

116 Molecular Dynamics (MD) simulations are an excellent choice to develop and analyze

117 models of such systems ${ }^{22}$.

118 In the present work, a set of hierarchical levels of structure are proposed for lipid

119 membranes, in line with those widely recognized for other biomolecular structures such

120 as proteins and DNA. Original MD simulations results of a large POPC lipid bilayer at

121 coarse grained (CG) resolution and of several macromolecules embedded in an atomistic

122 POPC membrane from a previous work are employed to illustrate the proposal. We aim

123 to introduce an original perspective for the description of lipid membranes, inspired by

124 what Franklin, Watson and Crick did with DNA and Kendrew did with proteins.

125 Hopefully, this new approach will contribute to understand better the behavior of

126 membrane models as well as of actual biological envelopes based on lipids. 


\section{2.- Results \& Discussion}

131 The different hierarchical levels of structure proposed to reinterpret membrane models

132 are described here in detail. As explained in the introduction, MD simulations of a large

133 POPC membrane at CG resolution as well as atomistic simulations of different

134 macromolecules embedded in a DOPC membrane taken from a previous work are used

135 to illustrate the new concepts. The large homogeneous membrane was chosen to show

136 the impact of the system size in some of the analyzed properties and to prove that even a

137 membrane with a single component exhibits the proposed levels of structure. The

138 atomistic simulations of more complex systems were employed to show the highest

139 level of structure, as explained later.

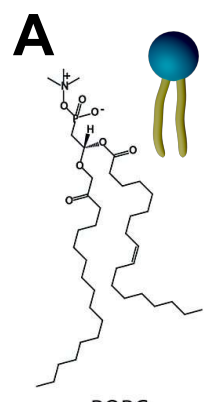

POPC

C
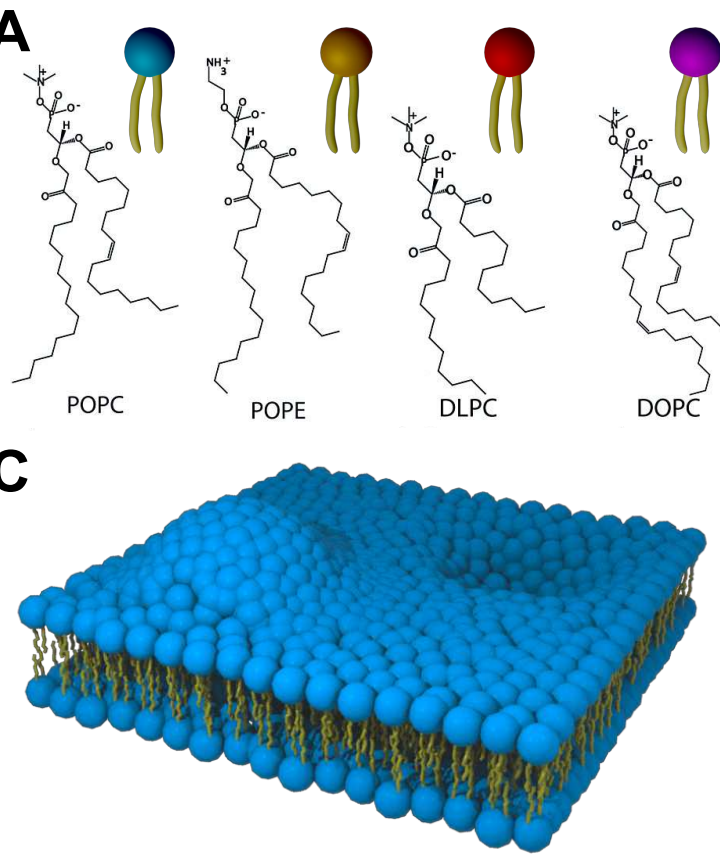

B
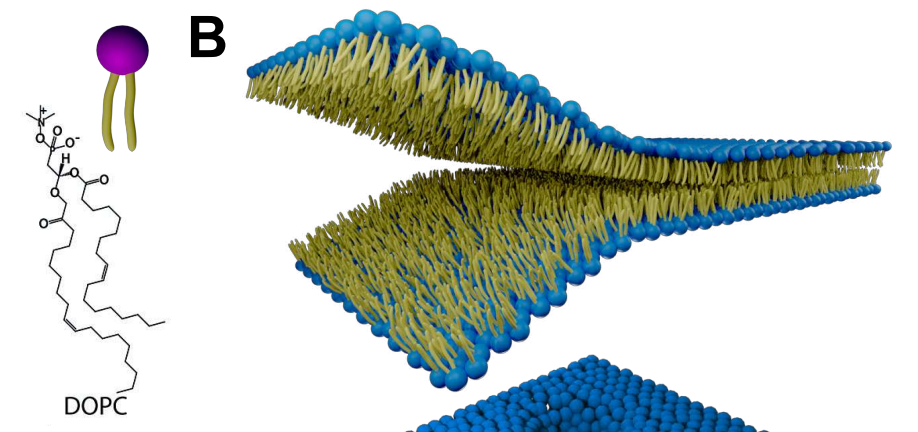

D
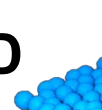

140

141

142

143

144

145

146

Figure 1.- Schematic representation of primary (A), secondary (B), tertiary (C) and quaternary (D) levels of structure of a lipid bilayer. Each color in A represents a different lipid type (just some examples, POPC, POPE, DLPC, DOPC are represented). See text for details. 
147 2.1.- The primary structure of the lipid bilayer: a "sequence" of thousands of non-

148 covalent basic units

149 In our analogy with the hierarchical representation of four structural layers of

150 organization in proteins and nucleic acids, the primary structure (Fig. 1A) of lipid

151 membranes is identified with the composition of each leaflet, that is different in

152 asymmetric bilayers. Variations in headgroups and aliphatic chains allows the existence

153 of more than 1,000 different lipid species just in a single eukaryotic cell ${ }^{23}$. As explained

154 in the introduction section, a number of infections and diseases have a serious impact on

155 the lipid composition of the host cells. This lipid composition can be employed as a

156 target for the actuation of the immune system and also for artificial therapeutic

157 treatments. Actually, all living organisms have a series of short, cationic and

158 amphipathic peptides known as Host Defense Peptides (HDPs) which are able to

159 recognize and disrupt pathological and pathogenic membranes based on their

160 composition ${ }^{24,25}$. These HDPs are an important part of the innate immune system. This

161 illustrates the huge importance of the lipid composition of biological membranes, and it

162 justifies that it is identified with the primary structure of the lipid bilayer. However,

163 there are clear discrepancies in this analogy with respect to the primary structure of

164 nucleic acids and proteins. The most obvious difference is that the lipids in a membrane

165 are not covalently bound, in contrast to the nucleotides in DNA or RNA and to amino

166 acids in proteins. Another evident difference is that lipid membranes are locally flat,

167 even though they can form closed quasi-spherical compartments with significant defects

168 or protuberances. As a consequence of the previous two features, membranes have

169 lipids diffusing throughout them, incorporate other molecules into their structure, fuse

170 with other membranes and exhibit a high ability to adapt to environmental changes by

171 altering their composition. Lipid membrane composition alterations take place as a 
172 response to almost any environmental change: $\mathrm{pH}$, temperature, diet, etc. ${ }^{26}$ This would

173 be equivalent to a protein or a nucleic acid altering its sequence as a response to an

174 external perturbation, which is not possible because their residues are joined by covalent

175 bonds. The high plasticity and flexibility of biological membranes allow them to

176 maintain their bioactivity despite the effect of external perturbations. Thus, within our

177 proposal, the primary sequence of membrane is simply the list of lipids in each leaflet.

178 2.2.- The secondary structure of the lipid bilayer: a Velcro strap more resistant than

179 helices or beta sheets

180 The secondary structure of DNA arises from the interaction between two polynucleotide

181 chains forming a double helix while in proteins it is defined by local and specific

182 interactions between amino acids leading to alpha-helices or beta-sheets, among other

183 patterns. The case of lipid bilayers is similar to DNA in the sense that they consist of

184 two monolayers facing each other as a Velcro strap whereas DNA has two

185 complementary chains interacting as a zipper (Fig 1B). This analogy is not only

186 structural but also with respect to the interactions. The local interactions (by H-bonds)

187 between two nucleotides in a DNA fragment is significantly stronger than the

188 hydrophobic (mainly van der Waals type) interaction between two lipids of different

189 leaflets in a membrane patch, however these latter interactions are highly cooperative-

190 exactly as in a Velcro strap- and altogether makes the interaction between monolayers

191 extremely strong.

192 This secondary structure is critical for the stability of lipid bilayers as lipid monolayers

193 do not exist in solution (although they are stable in a hydrophobic/hydrophilic

194 interphase). Again, there are differences between the two systems for this level of

195 structure, e.g. the interaction between leaflets taking place in two dimensions instead of 
196 the one-dimensional interaction between polynucleotide chains, and the fluidity of lipids

197 in a membrane compared to the fixed position of nucleotides in each DNA strand. As a

198 result of this level of structure, the two leaflets in a lipid bilayer are highly correlated to

199 each other. This is clearly illustrated in the topography of both monolayers as well as in

200 the field lines arising from the curvature, isocurvature and anisocurvature (see Methods)

201 vector fields (Fig. 2).
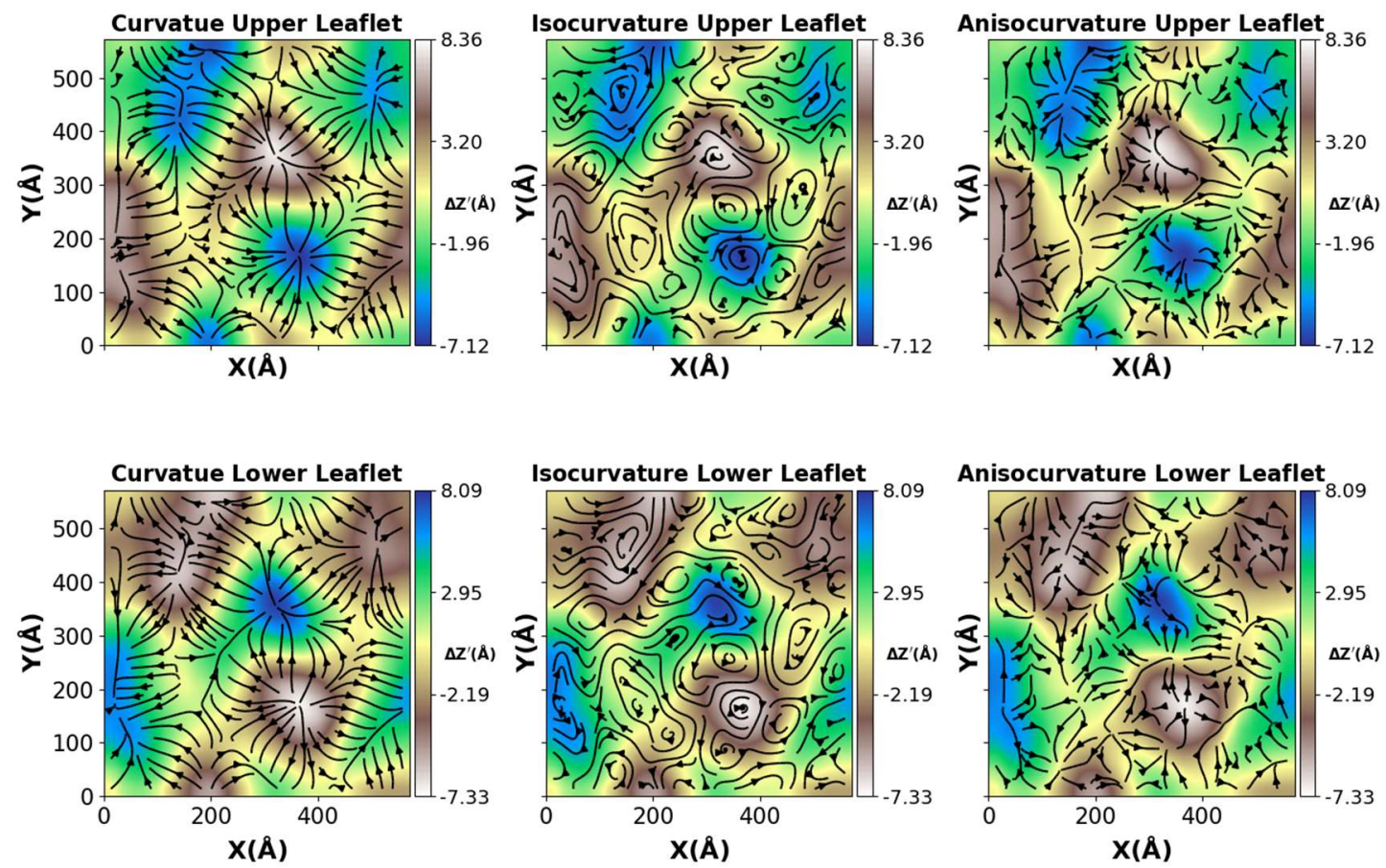

202

Figure 2.- Topographic heatmap and LRS vector fields for both leaflets (see labels on top of each plot). Image obtained from the average over the last ns of the trajectory. The topography maps exhibit clear hills and wells which projection in the XY plane spans a diameter of $\sim 15 \mathrm{~nm}$ with an amplitude of $\sim 0.8 \mathrm{~nm}$ in $Z$. The negative correlation between the two leaflets is evident. The field lines obtained from the curvature, isocurvature and anisocurvature vector fields are also clearly correlated with the topography of the membrane. The correlation/connection between different regions and the anti-complementarity between the two leaflets are also obvious in these vector field maps. The curvature shows field lines falling from the top of the hills and rising from the bottom of the wells, as expected. The isocurvature exhibits strong curls and long fields lines contouring regions of same elevation. The anisocurvature displays long and straighter field lines, with no curls at all, and with vector line sources and wells in the regions where the isocurvature

213 presents curls. 
217 structure of the lipid bilayer.

218 Higher levels of structure can be inferred from the coordinate behaviour of lipids,

219 leading to local domains that interact with each other (Fig. 1C). The definition of the

220 domains depends on the analyzed property. For instance, the topography of a membrane

221 patch may exhibit protuberances and valleys (Fig. 2), the same structure could also have regions of different thickness, and the lipid tails could be orientated in a coordinate way

223 forming patterns. Each of these properties would lead to different criteria to define

224 domains. The analysis proposed in the methodology section identifies different kind of

225 lipid domains as well as of interactions or couplings between them. The results

226 corresponding to the large POPC monolayer simulated using coarse grained resolution are presented and discussed in what follows.

\subsection{1.- Identification of domains}

229 Since we are interested in the structure of the bilayer, only the results for the last part of 230 the trajectory, which is assumed to be well equilibrated, will be shown here. As shown

231 above (Fig. 2), the diameter and amplitude of the membrane protuberances conditions

232 the vector fields arising from the LRS. Both the topography and these vector fields from 233 well-defined domains. The distribution of the angles between the surface normal vectors 234 and the $\mathrm{Z}$ axis of the simulation box exhibits a maximum at $\sim 0.06 \mathrm{rad}\left(3.4^{\circ}\right)$ and, as expected, they are indistinguishable for both leaflets (Fig. S1). The heatmaps obtained

236 from those angles show that larger angles correspond to the regions of maximum slope,

237 i.e., halfway between hills and wells (Fig. S1).

238 The vector fields arising from the lipid tail projection in the XY plane were determined

239 from the vectors connecting the PO4 group with the last bead of each tail (Fig. S2). 
240 Both tails behave in a similar way, with their corresponding vector fields emanating

241 from the highest points and converging in the lowest regions. This pattern was already

242 expected since the curvature itself conditions the orientation of the lipid tails in the

243 observed directions. The correlation between the topography and these vectors seems to

244 be slightly more marked for SN1 than for SN2, probably due to the perturbation induced

245 by the presence of the double bond in SN2. The lines arising from the vector field of the

246 lipid tail projection in the XY plane are antiparallel to those obtained from the curvature

$247\left(Z^{\prime}\right)$ vector field. It is also useful to measure the angle between the lipid tail vectors and

248 the $Z$ ' axis of the LRS. These distributions are equivalent to those shown by the surface

249 normal vectors (Fig. S1) but with the vectors pointing to the opposite direction (Fig.

250 S3). The corresponding heatmaps (Fig. S4) are less clear than those obtained from the

251 surface normal vectors, likely because the lipid bilayer is in fluid phase, but slighter

252 lower angles (i.e., with lower projection over the $Z$ ' axis of the LRS) can be observed

253 for the regions where the topographic slope is maximum.

254 The membrane thickness calculated considering the LRS exhibits a narrow normal-like

255 distribution centered around $3.9 \mathrm{~nm}$ (Fig. S5). The associated heatmap shows domains

256 of different thickness. The correlation between the thickness and the topography is not

257 evident, although the order of the lipid tails is expected to simultaneously depend on

258 both properties and so they are expected to have some connection.

\section{2.3.2.- Filtering non-redundant properties}

260 In general, it is clear that all the analyzed properties arise from the interaction between

261 groups of lipids and also that they define structural regions or domains in the lipid

262 bilayer that span several cells. These domains, as well as the interaction between them,

263 will be identified in the present work with the tertiary structure of the lipid bilayer, in 
analogy with that in nucleic acids and proteins arising from the relative spatial location

265 of nucleic acids and amino acids.

266 However, as stated above, the definition of the domains is not clear because those

267 defined by the topography may be different from those defined by the thickness or by

268 any other property. It is convenient to identify which properties are coupled to each

269 other, thus providing identical or cooperating domains, and which properties are

270 independent from the rest thus providing non redundant information. Next, a minimal

271 set of quantitative descriptors obtained from the properties calculated in the previous

272 section will be identified. This set of variables should be able to describe the structure

273 of the lipid bilayer without redundancy. The SVD factorization of the matrix containing

274 their z-score normalized values over all the grid cells and frames for the last $100 \mathrm{~ns}$ of

275 our trajectory will be employed for this aim (see methods section for details). As both

276 leaflets behave equivalently, the SVD analysis was separately carried out for each

277 leaflet and lipid tail, giving equivalent results (Figs S6-S9).

278 The explained variance per eigenvector is a step function with several groups of

279 properties contributing equally to the total variance (Figs S6-S9, panel A). This is a

280 consequence of the close connection between variables -such as the topography of the

281 membrane and the curvature, isocurvature and anisocurvature vectormaps- and their

282 cooperative behavior defining the membrane domains, which in this case would not be

283 defined by just one property but by the combination of several ones. Complementarily,

284 just 11 variables are required to explain the 95\% of the total variance (Figs S6-S9, panel

285 A). The $\mathrm{V}^{\mathrm{T}}$ matrix containing the eigenvector compounds in the space generated by the

286 properties used for the analysis, indicates too that several variables are coupled, since

287 they can be explained by almost identical eigenvectors. 
288 This coupling was quantified through the dot product between the column vectors

289 consisting of the normalized relevance matrix (Figs S6-S9, panel C). The results of

290 these dot products were then represented as a coupling matrix (Figs S6-S9, panel D). In

291 these matrices, it is clearly seen that the $\mathrm{X}$ and $\mathrm{Y}$ components of the isocurvature and

292 anisocurvature vectors are fully coupled to each other and the X and Y components of

293 the surface normal are strongly coupled to the $X^{\prime}$ and $Y^{\prime}$ components of the lipid tails,

294 respectively. These correlations were already expected after visually inspecting Fig. 2

295 and Fig S2, but finding them as a result can be considered as a validation of the method.

296 The previous information suggests that the minimum number of properties used to

297 efficiently characterize the lipid membrane can be reduced. Such set of properties could

298 be considered as an orthogonal basis of quantitative descriptors for the lipid bilayer. It is

299 interesting to observe the absolute lack of correlation between different properties,

300 indicating that they are independent and complementary to each other. For instance, the

301 topography of the membrane does not display any correlation with the $\mathrm{X}$ or $\mathrm{Y}$

302 component of either the iso or anisocurvature but does show it with the $\mathrm{Z}$ component of

303 the latter vector field. Some coupling is also observed with some of the other variables,

304 including the tail components in the LRS and the thickness. Interestingly, the

305 topography does not couple at all with the $\mathrm{Z}$ component of the normal. It is clearly seen

306 in Fig. 2 that the $\mathrm{X}$ and $\mathrm{Y}$ components of the normal vector are negligible in the maxima

307 and minima of the membrane protuberances, thus a clear correlation between the $\mathrm{Z}$

308 component of said vector and the relative $\mathrm{Z}$ position of the lipid heads was to be

309 expected. The reason for the observed lack of correlation is that it is only present at such

310 maxima and minima but, not in general, such as at intermediate positions which weigh

311 much more in the ensemble. For instance, the normal vectors at flat regions are also 
312 parallel to the $\mathrm{Z}$ axis of the global reference system, although the relative $\mathrm{Z}$ position of

313 the corresponding head groups is null in these cases.

314 The redundant properties of the previous analyses were detected based on their shape

315 similarities in the Coupling, Relevance and $\mathrm{V}^{\mathrm{T}}$ matrices. After removing them, the SVD

316 analysis was repeated with the following descriptors: the $\mathrm{X}$ and $\mathrm{Z}$ component of the

317 vectors consisting the LRS, the SN1 tail tilt, the membrane thickness and the $Z$,

318 component of the SN1 tail vectors in the LRS. Note that by not explicitly considering

319 some variables we are not actually eliminating them, but assuming that they can be well

320 represented by some other property. Thus, the labels of Fig. 3 will show which variables

321 are equivalent to each other in the proposed orthogonal model.

322 No redundant eigenvectors were observed in the cumulative variance plot (Fig. 3A), as

323 expected when all the properties considered for the analysis are independent from each

324 other. The final $\mathrm{V}^{\mathrm{T}}$, normalized relevance and coupling matrices (Fig. 3B-D) shows that

325 there are strong couplings between different variables, but no redundant pair of

326 properties are present. 

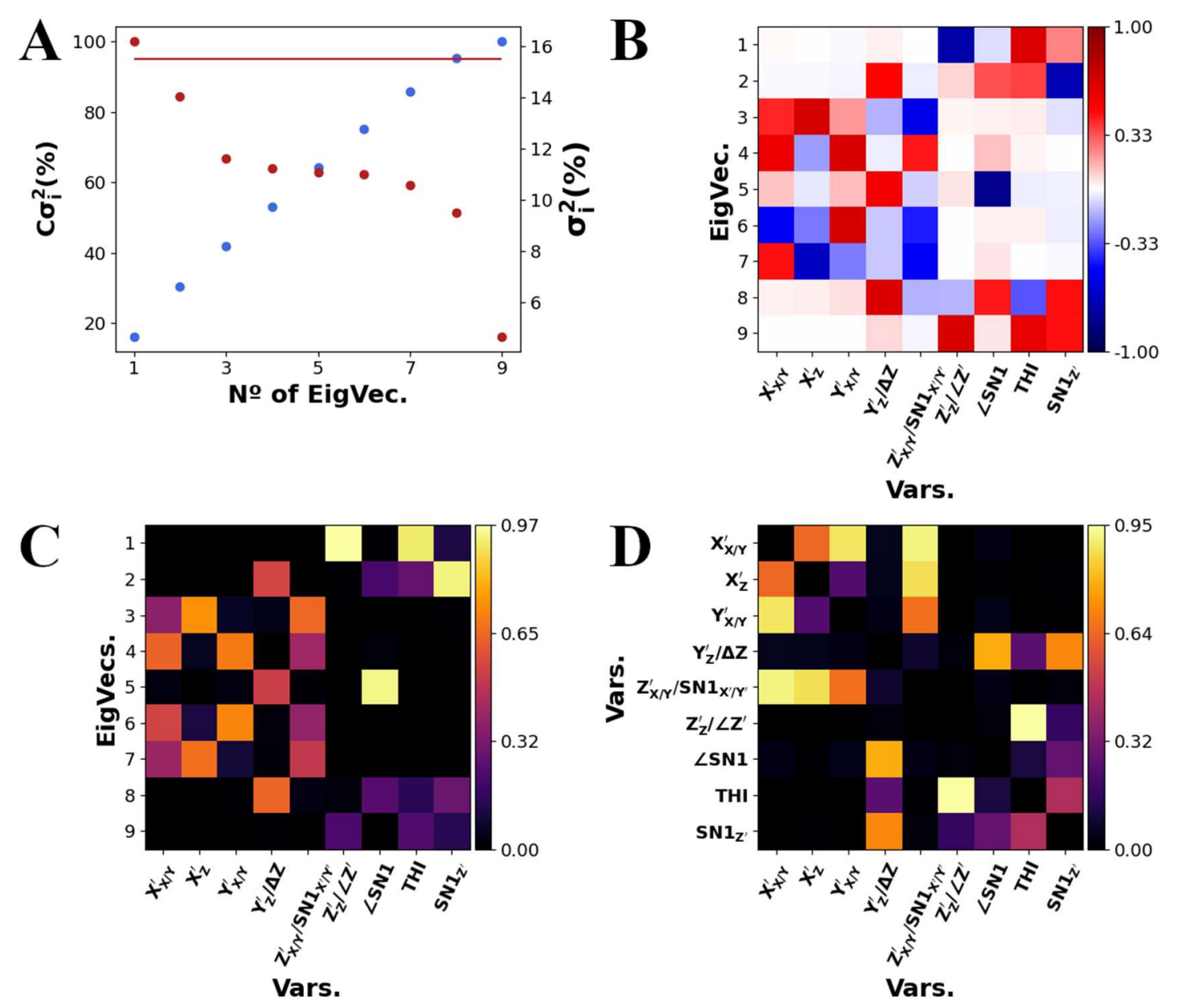

328 Figure 3.- Results for the SVD analysis performed on the nonredundant set of variables. Variance (red) and cumulative variance (blue), $95 \%$ explained marked with a red line $(\mathrm{A}) ; \mathrm{V}^{\mathrm{T}}$ matrix $(\mathrm{B})$; relevance matrix (C); coupling matrix (D). The $\mathrm{Z}$ component of the anisocurvature, and by extension the membrane topography, are coupled to tail-related parameters (tilt and $Z$ ' component) and the membrane's thickness, and completely uncoupled from the surface-defining vectors ( $X^{\prime}$ and $\left.Y^{\prime}\right)$ of the LRS. On the other hand, the $\mathrm{XY}$ components of the $Z^{\prime}$ LRS vector, and by extension the surface components of the lipid tails, are coupled entirely to the $X^{\prime}$ and $Y^{\prime}$ LRS vectors. Additionally, the $Z$ component of the $Z$ ' LRS vector, and by extension the membrane's curvature, are almost exclusively coupled to the membrane's thickness, which in turn couples to the $Z$ ' component of the lipid tails and the topography/anisocurvature vector field. Hence, we can infer that the membrane's topography is governed by how stretched the lipid tails are, influencing at the same time the membrane's thickness. That will create a concrete 3D shape in the membrane, with higher and lower regions which in turn will rule the orientation of the lipid tails, thus creating a feedback of influence.

342 From the initial set of 39 properties, considering both leaflets, around 9 quantitative descriptors resulted to be not superfluous. In analogy with the tertiary structure of proteins, the different domains interacting with each other-described by the set of nonredundant properties as shown in Fig. 3D- and their couplings can be associated to a tertiary structure in lipid bilayers. Thus, the coupling matrices (Fig. 3D) are expected to 
347 be a fingerprint of the tertiary structure of a lipid bilayer. In the present work a simple

348 homogenous POPC bilayer is analyzed. The size and shape of the domains defined by

349 the properties employed here, or by a different set of quantitative descriptors, as well as

350 the crossed interactions between such domains, could be totally different in a membrane

351 of different composition. Going further, the projection of the MD trajectory of the

352 membrane model on the different eigenvectors obtained from our analysis (Fig. S10)

353 could provide an alternative group of complementary domains defined by the collective

354 behavior of groups of lipids considering specific linear combinations of the quantitative

355 descriptors employed in our analysis.

356 2.4.- The quaternary structure of the lipid bilayer

357 The levels of structure described above are expected to be present in all membranes,

358 regardless of their composition, however the highest-order hierarchy remains to be

359 established. In nucleotides, the quaternary structure is related to the interaction with

360 different biomolecules which fold the double stranded DNA in different patterns.

361 Following the same philosophy, it is sensible to test the response of membranes to the

362 presence of macromolecules in their environment (Fig. 1D). 

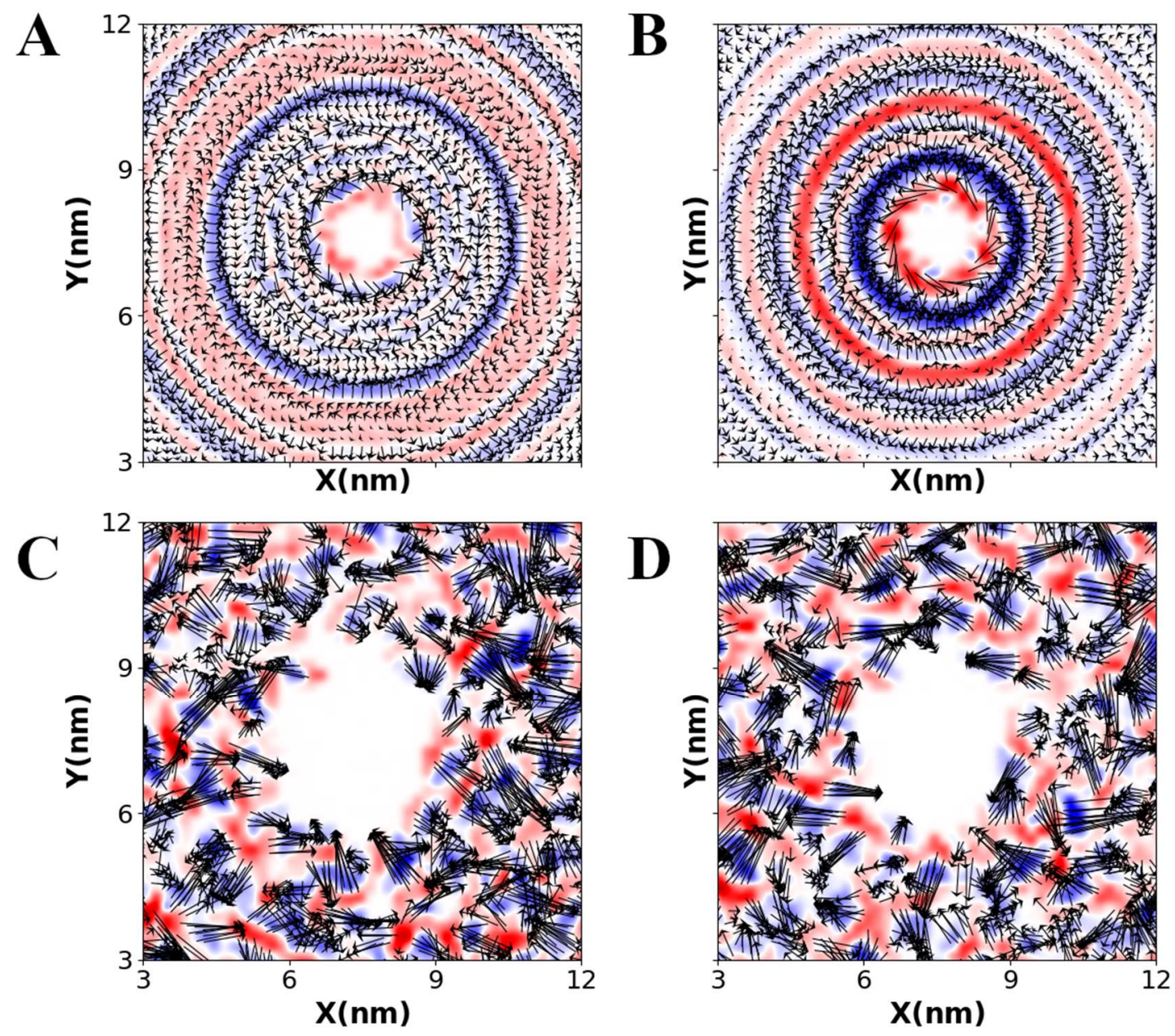

364 Figure 4.- Schematic representation of the quaternary structure in a DOPC lipid bilayer containing a carbon

365 nanotube $(A, B)$ and a $\beta$-barrel protein model $(C, D)$. The vector fields obtained from the orientation of the 366 lipid SN1 tail $(\mathrm{A}, \mathrm{C})$ and SN2 tail $(\mathrm{B}, \mathrm{D})$ in the upper leaflet are plotted together with the corresponding divergence represented in red-blue color gradient for divergence-convergence, respectively.

Taking advantage of previous work ${ }^{27}$ we test and compare the behaviour of an atomistic

370 DOPC membrane model when in the presence of a carbon nanotube (CNT) and a

371 simple $\beta$-barrel model (Fig. 4 and Fig. S11). Just by visual inspection, the influence of

372 the macromolecules on the lipid organization is remarkable and distinguishable. In both

373 cases the lipid tails become highly ordered around the macromolecule and they distort

374 the nominal kind of organization seen in previous sections. The CNT creates a series of

375 concentrical circular ripples around itself and generates a continuous pattern in the lipid tails. The divergence heatmap also displays the aforementioned rings, indicating that 
377 there will be circular regions defined by coordinate orientation of lipids. On the other

378 hand, the beta-barrel generates local clumps of lipids with the same orientation, but

379 there is no overall cohesion, contrary to the former case. Similarly, the divergence

380 heatmap also displays protein-induced lumps with no clear global organizational

381 pattern.

382 Thus, the case of the quaternary structure is defined straightforwardly from that of

383 proteins of nucleic acids: it is the response and interaction of lipid membranes to other

384 macromolecules, which is distinctive depending on the case.

386 Conclusions

387 A hierarchical set of structural levels is defined for lipid bilayers in analogy to the

388 primary, secondary, tertiary and quaternary structural levels of nucleic acids and

389 proteins. The primary structure is identified with the lipid composition of each leaflet,

390 the secondary structure is defined by the interaction between the two leaflets

391 conforming the membrane, the tertiary structure arises from the lipid domains obtained

392 from the analysis of the properties calculated throughout the membrane surface, as well

393 as from the interaction or coupling between such domains, and the quaternary structure

394 comes from the impact of macromolecules embedded in the lipid bilayers. The coupling

395 matrix proposed to identify the tertiary structure is expected to be a fingerprint

396 associated to each membrane composition since it describes how the orientation of the

397 lipid tails depends on the membrane topography, or how the membrane curvature

398 affects the bilayer thickness, among other connections. The impact of the presence of

399 macromolecules embedded in the membrane, corresponding to the quaternary structure,

400 is huge. The pattern of the lipid tails orientation seems to be highly specific around rigid 
structures such as carbon nanotubes and less ordered around more flexible structures.

402 This is a first approach to the definition of hierarchical structural levels for membrane models. Part of our treatment is based on a set of local quantitative descriptors, but different sets of properties could be employed with the same objective. Our proposal is illustrated with a simple POPC bilayer as well as by the simulation of several macromolecules embedded in a DOPC membrane. The connection of the domains

407 obtained from our analysis with biological activity is still unexplored. This new approach and analytical methods have a lot of room for improvement, but we hope it will impact in the understanding of membrane models as well as in the design of compartmentalized structures for new biotechnological applications.

\section{Methods}

\section{1.- Simulation parameters}

414 A flat-square membrane consisting of 5000 Martini 2.2 POPC lipids ${ }^{28}$ per leaflet was

415 built using the CHARMM-GUI Martini Maker ${ }^{29-31}$. The resulting bilayer, spanning 416 about $57 \mathrm{~nm}$ in the $\mathrm{X}$ and $\mathrm{Y}$ dimensions, was solvated using 115429 non-polarizable

417 Martini water particles, thus leading to a $57 \times 57 \times 8 \mathrm{~nm}^{3}$ box. No ions were added to the 418 system since the employed lipid is zwitterionic and so the membrane model is neutral in 419 the absence of additional charges. Periodic boundary conditions were applied to the 420 three spatial dimensions and the energy of the whole system was minimized for 5000 421 steps using the GROMACS 2020.4 molecular dynamics engine ${ }^{32,33}$ with a steepest 422 descent algorithm, a tolerance of $10 \mathrm{~kJ} \cdot \mathrm{mol}^{-1} \cdot \mathrm{nm}^{-1}$ and a step size of $0.01 \mathrm{~nm}$. The 423 simulation box was then equilibrated for $100 \mathrm{~ns}$ of molecular dynamics simulation in 424 five consecutive stages, where the time step was sequentially increased from 2 fs to 20 
425 fs using the leap-frog integrator. During this process the temperature was maintained at

$426310 \mathrm{~K}$ with a velocity rescale algorithm $\mathrm{m}^{34}$ and a coupling constant of $1 \mathrm{ps}$, while the

427 pressure was maintained at 1 atm with a Berendsen semi-isotropic barostat, ${ }^{35}$ a coupling

428 constant of 5 ps and a compressibility modulus of $3 \cdot 10^{-4} \operatorname{bar}^{-1}$. The electrostatic

429 interactions were calculated with the reaction-field method with a cutoff radius of 1.1

$430 \mathrm{~nm}$ and a dielectric constant of 15 beyond the cutoff. The van der Waals interactions

431 were calculated with a cutoff of $1.1 \mathrm{~nm}$.

432 After equilibration, an unrestrained 10- $\mu$ s-long MD production trajectory using a leap-

433 frog integrator with a timestep of $25 \mathrm{fs}$ was obtained. Removal of center of mass motion

434 was performed each 1000 steps, and position coordinates were written with the same

435 period (25 ps). Neighbor lists were obtained with the Verlet ${ }^{36,37}$ method and updated

436 every 20 steps. Temperature was maintained at $310 \mathrm{~K}$ with a velocity-rescale algorithm

437 and a coupling constant of 1 ps; while pressure was kept constant at 1 atm with a semi-

438 isotropic Parrinello-Rahman barostat, ${ }^{38}$ a coupling parameter of $12 \mathrm{ps}$ and a

439 compressibility modulus of $3 \cdot 10^{-4} \mathrm{bar}^{-1}$. Electrostatic interactions were calculated with

440 the reaction-field scheme, a cutoff radius of $1.1 \mathrm{~nm}$ and a dielectric constant of 15

441 beyond the given radius. van der Waals interactions were calculated with a cutoff of 1.1

$442 \mathrm{~nm}$.

\section{2.- Analysis}

444 Most of the analyses were carried with homemade scripts written in Python. The

445 recurrently used libraries were MDAnalysis ${ }^{39,40}$ to directly read trajectory files in

446 GROMACS format, Numba ${ }^{41}$ to facilitate high-performance parallelization schemes,

$447 \mathrm{NumPy}^{42}$ for general data handling and mathematical operations, and Matplotlib ${ }^{43}$ for

448 data representation. Visual depictions of the membrane were made using $\mathrm{VMD}^{44}$. 


\section{1.- Determination of local properties}

450 The analysis performed in this work relies on the discretization of the lipid membrane in

451 a square grid spanning the whole XY plane, with an average of $\boldsymbol{N}_{\boldsymbol{L}}$ lipid heads (PO4

452 groups) per cell. The coordinates of the grid cells are common for the two leaflets and

453 were kept constant over the entire segment of trajectory analyzed. A battery of different

454 properties was then determined for each cell and independently analyzed for each

455 leaflet.

456 In order to describe the dynamic behavior of the membrane, as well as local domains

457 and the connections between them, a local reference system (LRS) per grid cell as a

458 function of time was determined. The orthonormal vectors describing these LRS are: $(i)$

459 the normal to each cell, $\boldsymbol{Z}_{\boldsymbol{i}}^{\prime}(\boldsymbol{t})$; (ii) the normalized cross product between $\boldsymbol{Z}_{\boldsymbol{i}}^{\prime}(\boldsymbol{t})$ and the

460 gradient of its projection on the $\mathrm{Z}$ axis of the global reference system, $\boldsymbol{X}_{\boldsymbol{i}}^{\prime}(\boldsymbol{t})$; and (iii)

461 the cross product between the previous two vectors, $\boldsymbol{Y}_{\boldsymbol{i}}^{\prime}(\boldsymbol{t})$. The protocol used to define

462 these vectors is described in detail in the Supplementary Material (SM). The vector

463 fields arising from $\boldsymbol{Z}_{\boldsymbol{i}}^{\prime}(\boldsymbol{t}), \boldsymbol{X}_{\boldsymbol{i}}^{\prime}(\boldsymbol{t})$ and $\boldsymbol{Y}_{\boldsymbol{i}}^{\prime}(\boldsymbol{t})$ will be identified with the curvature, the

464 isocurvature and the anisocurvature, respectively, of the lipid bilayer. By using these

465 LRS it is possible to determine structural properties such as membrane thickness or tail

466 orientation while accounting for local deformations of the lipid bilayer. Such

467 deformations might be ignored in small membranes, but they are significant in patches

468 of several tens of nm long per edge, as it will be shown later.

469 The set of properties analyzed in the present paper can be computed with different time

470 and size resolution. The size resolution is given by the number of lipids per grid cell

$471\left(N_{L}\right)$ while the time resolution $(\Delta t)$ depends on the frequency of the fluctuations

472 observed for each property. Different values of these two parameters were tested in our 
code and after several tests we decided to present the results obtained with $N_{L}=10$ and a

474 moving average window of $\Delta \mathrm{t}=1 \mathrm{~ns}$. These values allow observing significant changes

475 in the quantitative properties computed for the lipid bilayer, with negligible noise and

476 optimizing the computational resources employed. Using these parameters, the

477 topography of the membrane was determined as the average $\mathrm{Z}$ coordinate of the PO4

478 groups per grid cell. The local membrane curvature was quantified as the projection of

$479 Z_{i}^{\prime}(t)$ on the $\mathrm{Z}$ axis. The thickness of the membrane and the orientation of the lipid tails

480 were determined using the LRS. The thickness is given by the average difference

481 between the position of PO4 groups along the local $\boldsymbol{Z}_{\boldsymbol{i}}^{\prime}(\boldsymbol{t})$ axes, while the orientation of

482 the tails is the difference between the coordinates of the PO4 and last beads of each tail 483 expressed in the LRS.

\section{$484 \quad$ 2.2.- Global analysis of the lipid bilayer}

485 Once the previously described properties are computed for each grid cell, time dependent local domains could be directly observed by plotting them in suitable representations (see results section and SM). A Singular Value Decomposition (SVD) analysis is then performed to identify a set of independent properties associated to the membrane structure as well as to quantify the coupling between the different studied properties (see SM for a detailed description). Each of the three components of the previously defined vectorial magnitudes were independently treated as scalar properties for this analysis. 
497 1. Watson, J. D. \& Crick, F. H. C. Molecular structure of nucleic acids: A structure

498 for deoxyribose nucleic acid. Nature 171, 737-738 (1953).

499 2. Franklin, R. E. \& Gosling, R. G. Evidence for 2-chain Helix in crystalline

$500 \quad$ structure of sodium deoxyribonucleate. Nature 172, 156-157 (1953).

501 3. Kendrew, J. C. The three-dimensional structure of a protein molecule. Sci. Am.

502 205, 96-110 (1961).

503 4. Kendrew, J. C. et al. A three-dimensional model of the myoglobin molecule $504 \quad$ obtained by x-ray analysis. Nature 181, 662-666 (1958).

505 5. Singer, S. J. \& Nicolson, G. L. The fluid mosaic model of the structure of cell 506 membranes. Science (80-. ). 175, 720-731 (1972).

507 6. Kusumi, A., Suzuki, K. G. N., Kasai, R. S., Ritchie, K. \& Fujiwara, T. K. 508 Hierarchical mesoscale domain organization of the plasma membrane. Trends $509 \quad$ Biochem. Sci. 36, 604-615 (2011).

510 7. Alberts, B. et al. Molecular Biology of the Cell. (Garland Science, 2014).

5118 8. Nussinov, R., Tsai, C. J. \& Jang, H. Ras assemblies and signaling at the 512 membrane. Curr. Opin. Struct. Biol. 62, 140-148 (2020).

513 9. Corradi, V. et al. Emerging Diversity in Lipid-Protein Interactions. Chem. Rev. $514 \quad 119,5775-5848(2019)$.

515 10. Stieger, B., Steiger, J. \& Locher, K. P. Membrane lipids and transporter function. 516 Biochim. Biophys. Acta-Mol. Basis Dis. 1867, 166079 (2021).

517 11. Sens, P. \& Plastino, J. Membrane tension and cytoskeleton organization in cell 
motility. J. Phys. Condens. Matter 27, 273103 (2015).

519 12. Kielian, M. \& Jungerwirth, S. Mechanisms of Enveloped virus entry into cells. Mol. Biol. Med. 7, 17-31 (1990).

13. Dimitrov, D. S. Virus entry: Molecular mechanisms and biomedical applications. Nat. Rev. Microbiol. 2, 109-122 (2004).

14. Münz, C. The autophagic machinery in viral exocytosis. Front. Microbiol. 8, 269 (2017).

15. Garcia-Fandino, R. \& Piñeiro, Á. Delving Into the Origin of Destructive Inflammation in COVID-19: A Betrayal of Natural Host Defense Peptides? Front. Immunol. 11, 3532 (2021).

16. Szlasa, W., Zendran, I., Zalesińska, A., Tarek, M. \& Kulbacka, J. Lipid composition of the cancer cell membrane. J. Bioenerg. Biomembr. 52, 321-342 (2020).

17. Alves, A. C., Ribeiro, D., Nunes, C. \& Reis, S. Biophysics in cancer: The relevance of drug-membrane interaction studies. Biochim. Biophys. ActaBiomembr. 1858, 2231-2244 (2016).

18. Glukhov, E., Stark, M., Burrows, L. L. \& Deber, C. M. Basis for selectivity of cationic antimicrobial peptides for bacterial versus mammalian membranes. $J$. Biol. Chem. 280, 33960-33967 (2005).

19. Sohlenkamp, C. \& Geiger, O. Bacterial membrane lipids: Diversity in structures and pathways. FEMS Microbiol. Rev. 40, 133-159 (2015). inflammatory diseases. Int. J. Mol. Sci. 21, 3074 (2020). 
541 21. Leitinger, N. The role of phospholipid oxidation products in inflammatory and autoimmune diseases: Evidence from animal models and in humans. Subcell. Biochem. 49, 325-350 (2008).

22. Moradi, S., Nowroozi, A. \& Shahlaei, M. Shedding light on the structural properties of lipid bilayers using molecular dynamics simulation: a review study. RSC Advances vol. 9 4644-4658 (2019).

23. Sud, M. et al. LMSD: LIPID MAPS structure database. Nucleic Acids Res. 35, D527-D532 (2007).

24. Magana, M. et al. The value of antimicrobial peptides in the age of resistance. Lancet Infect. Dis. 20, e216-e230 (2020).

25. Huan, Y., Kong, Q., Mou, H. \& Yi, H. Antimicrobial Peptides: Classification, Design, Application and Research Progress in Multiple Fields. Frontiers in Microbiology vol. 11582779 (2020).

26. Ernst, R., Ballweg, S. \& Levental, I. Cellular mechanisms of physicochemical membrane homeostasis. Current Opinion in Cell Biology vol. 53 44-51 (2018).

27. Garcia-Fandiño, R., Piñeiro, Á., Trick, J. L. \& Sansom, M. S. P. Lipid Bilayer Membrane Perturbation by Embedded Nanopores: A Simulation Study. ACS Nano 10, 3693-3701 (2016).

28. De Jong, D. H. et al. Improved parameters for the martini coarse-grained protein force field. J. Chem. Theory Comput. 9, 687-697 (2013).

29. Jo, S., Kim, T., Iyer, V. G. \& Im, W. CHARMM-GUI: A web-based graphical user interface for CHARMM. J. Comput. Chem. 29, 1859-1865 (2008). 
AMBER, OpenMM, and CHARMM/OpenMM Simulations Using the CHARMM36 Additive Force Field. J. Chem. Theory Comput. 12, 405-413 (2016).

31. Hsu, P. C. et al. Charmm-gui martini maker for modeling and simulation of complex bacterial membranes with lipopolysaccharides. J. Comput. Chem. 38, 2354-2363 (2017).

32. Lindahl, Abraham, Hess \& Spoel, van der. GROMACS 2020.4 Manual. (2020) doi:10.5281/ZENODO.4054996.

33. Abraham, M. J. et al. Gromacs: High performance molecular simulations through multi-level parallelism from laptops to supercomputers. SoftwareX 1-2, 19-25 (2015).

34. Bussi, G., Donadio, D. \& Parrinello, M. Canonical sampling through velocity rescaling. J. Chem. Phys. 126, 014101 (2007).

35. Berendsen, H. J. C., Postma, J. P. M., Van Gunsteren, W. F., Dinola, A. \& Haak, J. R. Molecular dynamics with coupling to an external bath. J. Chem. Phys. 81, 3684-3690 (1984).

36. Verlet, L. Computer 'experiments' on classical fluids. I. Thermodynamical properties of Lennard-Jones molecules. Phys. Rev. 159, 98-103 (1967).

37. Chialvo, A. A. \& Debenedetti, P. G. An automated Verlet neighbor list algorithm with a multiple time-step approach for the simulation of large systems. Comput. Phys. Commun. 70, 467-477 (1992).

38. Parrinello, M. \& Rahman, A. Polymorphic transitions in single crystals: A new molecular dynamics method. J. Appl. Phys. 52, 7182-7190 (1981). 
587

588

589

590

591

592

593

594

595

596

597

598

599

600

601

602

603

604

605

606

607

608

609

39. Gowers, R. et al. MDAnalysis: A Python Package for the Rapid Analysis of Molecular Dynamics Simulations. in Proceedings of the 15th Python in Science Conference 98-105 (2016). doi:10.25080/majora-629e541a-00e.

40. Michaud-Agrawal, N., Denning, E. J., Woolf, T. B. \& Beckstein, O. MDAnalysis: A toolkit for the analysis of molecular dynamics simulations. $J$. Comput. Chem. 32, 2319-2327 (2011).

41. Lam, S. K., Pitrou, A. \& Seibert, S. Numba: A LLVM-based Python JIT Compiler. in Proceedings of the Second Workshop on the LLVM Compiler Infrastructure in HPC - LLVM '15 (ACM Press).

42. Harris, C. R. et al. Array programming with NumPy. Nature 585, 357-362 (2020).

43. Hunter, J. D. Matplotlib: A 2D graphics environment. Comput. Sci. Eng. 9, 9095 (2007).

44. Humphrey, W., Dalke, A. \& Schulten, K. VMD: Visual molecular dynamics. J. Mol. Graph. 14, 33-38 (1996).

\section{Acknowledgements}

This work was supported by the Spanish Agencia Estatal de Investigación (AEI) and the ERDF (RTI2018-098795-A-I00, and PID2019-111327GB-I00), by Xunta de Galicia and the ERDF (ED431F 2020/05 and Centro singular de investigación de Galicia accreditation 2016-2019, ED431G/09), and by Fundação para a Ciência e Tecnologia (FCT) funds and FEDER European funds (POCI-01-0145-30579). R.G.-F. thanks Ministerio de Ciencia, Innovación y Universidades for a "Ramón y Cajal” contract (RYC- 
610 2016-20335). A. B.-G thanks Xunta de Galicia for a "Doutoramento Industrial" contract

611 (06_IN606D_2021_2600276). All calculations were carried out at the Centro de 612 Supercomputación de Galicia (CESGA).

613

614 Contributions

615 Á.P., and R. G-F. conceived the study. A.B. performed the simulations and analysis. All 616 authors discussed the results, contributed to write the manuscript.

617

618 Corresponding authors

619 Correspondence to Rebeca Garcia-Fandino or Ángel Piñeiro.

620

621 Competing interests

622 The authors declare no competing interests.

623

624 


\section{Supplementary Files}

This is a list of supplementary files associated with this preprint. Click to download.

- SM20220122.pdf 